\title{
Bevacizumab for glioblastoma
}

This article was published in the following Dove Press journal:

Therapeutics and Clinical Risk Management

I December 2015

Number of times this article has been viewed

\section{Yoshitaka Narita}

Department of Neurosurgery and Neuro-Oncology, National Cancer Center Hospital, Tokyo, Japan
Correspondence: Yoshitaka Narita Department of Neurosurgery and Neuro-Oncology, National Cancer Center Hospital, 5-I-I, Tsukiji, Chuo-ku, Tokyo, 104-0045, Japan

Tel +8I 33542 25II

Fax +8I 33542255

Email yonarita@ncc.go.jp
Abstract: Individuals with glioblastoma are often characterized by older age, advanced neurologic manifestations at the primary stage, and unresectable tumors, and these factors are associated with poor treatment outcomes. Administration of bevacizumab (BV, Avastin $\left.{ }^{\circledR}\right)$ promotes tumor regression and improves cerebral edema, and is expected to improve neurologic findings in many patients with malignant gliomas, including glioblastoma. Although the addition of BV to the conventional standard therapy (chemoradiotherapy with temozolomide) for newly diagnosed glioblastoma prolonged the progression-free survival time and the performance status of patients, it failed to extend overall survival time. However, more than $50 \%$ of glioblastoma patients show Karnofsky performance status $\leq 70$ at initial presentation; therefore, BV should be used to improve or maintain their performance status as an initial treatment. Most of the adverse events of BV, except hypertension and proteinuria, occur as complications of glioblastoma, and explanation of the advantages and disadvantages of BV administration to patients is important. Herein, the efficacy, safety, and challenges of using BV for treating glioblastoma were reviewed.

Keywords: bevacizumab, glioblastoma, glioma, adverse events

\section{Introduction}

Glioblastoma has one of the worst prognoses among the various carcinomas, with a 5-year survival rate of $\sim 10 \%$. ${ }^{1}$ Standard therapy for glioblastoma is to remove as much tumor as possible without compromising neurologic function, followed by maintenance with concomitant temozolomide (TMZ) and radiation therapy (RT, focal radiation with 60 gray). The results of a Phase III study conducted by the European Organization for Research and Treatment of Cancer (EORTC) and the National Cancer Institute for Canada (NCIC) comparing RT + TMZ to RT alone in patients with primary glioblastoma showed that overall survival (OS) was significantly longer in the RT + TMZ group (14.6 months) than in the RT alone group (12.1 months). ${ }^{2,3}$

Most patients are diagnosed with glioblastoma within 3 months after symptom onset. One therapeutic challenge is that these patients show neurologic manifestations and have low performance status (PS) at initial presentation. At onset, Karnofsky PS (KPS) scores of 100/90 and 80 were found in $25 \%$ and $19 \%$ of patients, respectively, and KPS $\leq 70$ was found in $50 \%$ of patients. Therefore, PS in more than $50 \%$ of patients is already low during the primary diagnostic stage. ${ }^{1}$

Glioblastoma promotes secretion of vascular endothelial growth factor (VEGF), which induces vascularization and increases capillary permeability of the blood-brain barrier, subsequently causing extracellular edema. ${ }^{4,5}$ Bevacizumab (BV) (Avastin ${ }^{\circledR}$; Hoffman-La Roche Ltd., Basel, Switzerland) is a humanized monoclonal antibody targeting VEGF and was first approved in the US for glioblastoma in 2009. ${ }^{6}$ Blocking the VEGF pathway restores the abnormal tumor vasculature to a more normal state, reducing vascular permeability and the regional cerebral blood volume around 
the tumor ${ }^{7}$ in addition to decreasing peritumoral edema. Traditionally, steroids have been used to control brain edema in patients with brain tumors and $\sim 30 \%-70 \%$ of patients who received $\mathrm{BV}$ were reported to reduce their steroid doses. ${ }^{8,9}$ $\mathrm{BV}$ is a useful drug for glioblastoma patients especially those with poor PS as BV markedly reduces cerebral edema and improves PS. ${ }^{10,11}$ In this article, the efficacy and adverse events of BV are reviewed.

\section{Bevacizumab for newly-diagnosed glioblastoma}

A Phase III comparative study of $\mathrm{RT}+\mathrm{TMZ}+$ placebo versus $\mathrm{RT}+\mathrm{TMZ}+\mathrm{BV}$ was conducted to verify the additional effect of BV versus TMZ alone in patients with newly-diagnosed glioblastoma. The AVAglio study ${ }^{8}$ was conducted in European and Asian countries and in the US, and the RTOG0825 study ${ }^{12}$ was conducted mainly in the US. Progression-free survival (PFS) was 10.6 months in the BV group compared to 6.2 months in the placebo group in the AVAglio study, showing a significant PFS prolongation in the BV group (hazard ratio $[\mathrm{HR}] 0.64, P<0.001$ ). Although PFS in the BV group was also prolonged in the RTOG0825 study (7.3 vs 10.3 months) (HR $0.79, P=0.007$ ), the prespecified difference $(P<0.004)$ was not satisfied. In addition, no significant difference was observed in OS between groups in either the AVAglio study (16.7 vs 16.8 months) or the RTOG0825 study (16.1 vs 15.7 months). In both studies, the aim had been to prove the advantage of combination therapy with TMZ and BV; however, both clinical trials failed to prove this from the perspective of prolonged survival.

Patients with insufficient tissue at biopsy for genetic analysis were excluded from the RTOG0825 study. The treatments (BV or placebo) were assigned to patients in such a way that neither the doctors nor the patients were aware of which treatment was assigned to which patient. After recurrence, the trial became open, and BV was administered if needed. In the RTOG0825 study, patients were assigned to groups based on the expression level of nine genes thought to be prognostic factors. ${ }^{13} \mathrm{BV}$ was administered to $30 \%$ and $40 \%$ of patients in the placebo group after recurrence in the AVAglio study and in the RTOG0825 study, respectively. Thus, the crossover effect may have contributed to nondifferences in survival between the groups.

Most clinical studies are conducted in patients with KPS $\geq 60-70$. In fact, patients with KPS $\geq 90$ accounted for $70 \%$ of the study population in the AVAglio study. However, patients with KPS $\geq 90$ accounted for only $20 \%-30 \%$ of all patients with newly-diagnosed glioblastoma in clinical situations, as mentioned previously. ${ }^{1}$ Therefore, it is important to recognize the extreme difference in patients between clinical studies and the clinical setting. The HR for survival in the AVAglio study was 0.95 (95\% confidence interval [CI]: 0.77-1.19) in patients with an Eastern Cooperative Oncology Group (ECOG) PS score of 0 (KPS equivalent, 90-100), while it was 0.84 (95\% CI: 0.69-1.04) in patients with ECOG PS score of 1-2 (KPS 60-80), ${ }^{8}$ suggesting that among patients with low PS at the primary stage, OS might be prolonged by combined use of BV. In patients from this same study who did not receive post-study treatment due to low PS or other reasons, OS was 11.6 months in the $\mathrm{RT}+\mathrm{TMZ}+\mathrm{BV}$ group, while it was reported as 8.0 months in the $\mathrm{RT}+\mathrm{TMZ}$ group $(P=0.01)$.

In the AVAglio study, the time maintaining KPS $\geq 70$ was 6 months in the placebo group, while it was significantly prolonged to 10 months in the BV group, ${ }^{8}$ suggesting another advantage of BV administration at the primary stage.

In both studies, health-related quality of life (HRQOL) was evaluated using EORTC QLQ-C30/BN-20. Global health status, physical functioning, social functioning, motor dysfunction, and communication deficits were evaluated in the AVAglio study, and no $\geq 10$-point change during the time before recurrence was observed in either group. ${ }^{14}$ In the meantime, deterioration-free survival, which was defined as avoiding quality of life reductions of more than 10 points, recurrence, and death, was significantly prolonged in the RT + $\mathrm{TMZ}+\mathrm{BV}$ group. However, only global health status, social functioning, and communication deficit recurrence were prolonged in the $\mathrm{RT}+\mathrm{TMZ}+\mathrm{BV}$ group in the evaluation of time to deterioration, excluding recurrence and death from the events. In particular, the report indicated a lowered QOL score at the time of recurrence as an important finding. ${ }^{14}$

On the other hand, the results of the RTOG0825 study indicated the opposite result seen in the AVAglio study, showing a decrease of cognitive function ${ }^{15}$ as well as HRQOL score in the RT + TMZ + BV group until week 34 from baseline. ${ }^{16}$ The end point of HRQOL was defined as the time to overt deterioration in the AVAglio study, while it was defined as differences in score during a certain period after treatment initiation in the RTOG0825 study. As shown earlier, in addition to the difference in analytical methods between studies, it is important to note that the difference in survey response rate (AVAglio study: 80\% vs RTOG0825: 60\%) may affect the results. Therefore, it is still unclear if BV improves QOL in patients with newly-diagnosed glioblastoma.

Glioblastoma is classified into proneural, mesenchymal, and proliferative types by molecular markers 
based on microarray analysis. ${ }^{17}$ Only in patients with the proneural type tumor with IDH1 wild type was OS in the $\mathrm{RT}+\mathrm{TMZ}+\mathrm{BV}$ group significantly prolonged compared to $\mathrm{OS}$ in the RT + TMZ + placebo group (17.1 vs 12.8 months, HR 0.63, $P=0.045) .{ }^{18}$ However, no difference in OS was observed, while PFS was prolonged in the mesenchymal type with a high expression level of VEGF. Presence/absence of MGMT promoter methylation did not have any effect on the efficacy of BV. Further investigation is necessary for elucidating biomarkers indicating which gliomas will respond to $\mathrm{BV}$.

Administration of BV to patients with glioblastoma has been of concern because it can cause an increase of infiltration while it suppresses enlargement of the tumor; however, prospective examination of changes in the images in the AVAglio study revealed that BV did not cause any difference in the pattern of tumor invasion at the time of recurrence. ${ }^{19}$ Diffuse type is found in $61 \%$ of patients with glioblastoma at initial presentation. OS of patients with nondiffuse type was reported as 20.1 and 18.4 months in the RT + TMZ + BV group and the $\mathrm{RT}+\mathrm{TMZ}+$ placebo group, respectively, indicating prolongation of survival time in patients with nondiffuse type ( $P=0.03$, HR 0.76). Moreover, no difference in survival time was observed between groups even though patients with nondiffuse type at initial presentation turned out to have diffuse type at the time of recurrence. ${ }^{19}$

\section{Bevacizumab for recurrent glioblastoma}

The efficacy of BV monotherapy was demonstrated when patients in the RT + TMZ group experienced a recurrence. ${ }^{6,20-26}$ Before the $\mathrm{BV}$ era, PFS, the 6-month PFS rate, OS, and 1-year OS in patients with recurrent glioblastoma were reported as $\sim 2-3$ months, $9 \%-28 \%, \sim 6$ months, and $14 \%-32 \%$, respectively. ${ }^{25} \mathrm{PFS}, 6$-month PFS rate, and OS after BV therapy in patients with recurrent glioblastoma were reported as 2-4 months, 20\%-50\%, and 7-12 months, respectively. ${ }^{27}$

In the Brain study, the first large-scale study conducted in the US, BV monotherapy for patients with recurrent glioblastoma resulted in a response rate of $28.2 \%$, a 6-month PFS rate of $42.6 \%$, and median OS of 9.2 months. ${ }^{21}$ No difference in cognitive function during the time to recurrence due to administration of BV was reported. ${ }^{28}$

Previous research has indicated that the efficacy of BV monotherapy correlates with a patient's PS, and the effect of BV was less in patients with KPS $<70 .{ }^{29,30}$ However, patients with very low KPS were also reported to improve function and quality of life and be able to continue treatment at the outpatient clinic. ${ }^{11}$

The half-life of BV is $\sim 3$ weeks, and $10 \mathrm{mg} / \mathrm{kg}$ or $15 \mathrm{mg} / \mathrm{kg}$ of BV is administered every 2 weeks or 3 weeks, respectively; in other words, $5 \mathrm{mg} / \mathrm{kg}$ of BV is administered per week. No differences in PFS and response rate were reported even when low-dose BV (5 mg/kg/3 weeks) was administered. ${ }^{26}$ More favorable outcomes were reported when low-dose BV (less than $5 \mathrm{mg} / \mathrm{kg} /$ week) was administered. ${ }^{31,32}$ The optimal dose of BV for glioma patients should be determined.

The BELOB study was a randomized Phase II comparative study and the first study to compare CCNU (lomustine) to $\mathrm{BV}$ versus $\mathrm{CCNU}$ or $\mathrm{CCNU}+\mathrm{BV} .{ }^{33} \mathrm{OS}$ rate at 9 months in the CCNU group (50 cases), the BV group (46 cases), and the CCNU ( $90 \mathrm{mg} / \mathrm{m}^{2}$ or $\left.110 \mathrm{mg} / \mathrm{m}^{2}\right)+$ BV group (52 cases) was $43 \%, 38 \%$, and $59 \%$, respectively, and OS was 8,8 , and 11 months, respectively. PFS rate at 6 months was $43 \%$, $38 \%$, and $59 \%$, and PFS was 1, 3, and 4 months, respectively. Response rate in the BV monotherapy group was higher than that in the CCNU monotherapy group. Although no difference in OS was observed among groups, OS was prolonged in the $\mathrm{CCNU}+\mathrm{BV}$ group compared to the CCNU or BV monotherapy group. In this study, BV monotherapy failed to show superiority to CCNU monotherapy. These results lead to a randomized Phase III study comparing CCNU alone to $\mathrm{CCNU}+\mathrm{BV}$, conducted using CCNU as a standard therapy drug for patients with first recurrent glioblastoma.

Various clinical studies of BV + concomitant $\operatorname{drug}(\mathrm{s})$ have been conducted in patients with recurrent glioblastoma. However, promising results have not yet been obtained from combinations of BV with $\mathrm{TMZ},{ }^{34,35} \mathrm{BCNU},{ }^{36}$ fotemustine, ${ }^{37,38}$ irinotecan, ${ }^{21,39}$ or carboplatin. ${ }^{40-42}$

$\mathrm{BV}$ is reported to be effective for treating radiation necrosis. ${ }^{43}$ Re-irradiation of patients with glioblastoma who received radiation therapy as primary therapy may lead to radiation necrosis as a serious adverse event. However, a combination of $\mathrm{BV}$ and re-irradiation was reported to be effective. $^{44-47}$

Clinical studies of various drugs have been conducted in patients with recurrent glioblastoma; however, thus far an effective therapy has been elusive. No difference between electric field therapy using Novo TTF and other active chemotherapies was reported; ${ }^{48}$ thus, Novo TTF was approved as a medical device. PFS was reported to be $\sim 4$ months after administration of $\mathrm{BV}$ at the first recurrence and the second or third recurrence. ${ }^{49,50}$ Some researchers suggest administering BV with palliative intent to patients with recurrent glioblastoma. 
Rapid enlargement of tumor or cerebral edema (rebound) after termination of BV has been reported, ${ }^{51}$ thus, administration of $\mathrm{BV}$ beyond progression is considered useful even after recurrences. In fact, the results of a randomized comparative study after recurrence in patients with colorectal cancer demonstrated prolonged survival in the BV-continuing group. ${ }^{52,53}$ Reardon et al retrospectively examined patients who experienced recurrence after administration of BV. OS in patients without treatment, patients treated with a non-BV regimen, and patients who continued BV therapy was $1.5,4.0$, and 5.9 months, respectively (HR $0.64, P=0.04$ ), indicating efficacy of "Beyond PD (BPD)" therapy even in patients with glioblastoma. ${ }^{54}$ However, BV continuation did not have any significant effect on PFS, or OS, or any clinical improvement in comparisons of BV-continuing groups to BV-ceasing groups ${ }^{55}$ conducted in patients with a first recurrent glioblastoma after a comparative study of BV vs BV + carboplatin. ${ }^{40}$ Currently, a BPD study is being conducted in patients who were enrolled in the AVAglio study, assigning those patients after recurrence into a BV group or a placebo group.

\section{Adverse events of bevacizumab}

Serious adverse events from BV include hypertension, arterial and venous thrombosis, intracerebral hemorrhage including tumor hemorrhage, proteinuria, delayed wound healing, and gastrointestinal perforation. ${ }^{27}$ The rate of these grade 3/4 adverse events according to the National Cancer Institute Common Terminology Criteria for Adverse Events version 3.0 for newly-diagnosed patients treated with $\mathrm{TMZ}+\mathrm{BV}^{8,12}$ and recurrent patients treated with single $\mathrm{BV}^{21,23-25}$ were reported as $1 \%-11 \% / 4 \%-16 \%$ (hypertension), $2 \%-8 \% / 3 \%-6 \%$ (arterial and venous thrombosis), $1 \%-2 \% / 0 \%$ (intracerebral hemorrhage), 5\%/0\%-3\% (proteinuria), 1\%-3\%/0\%-2\% (delayed wound healing), and $1 \% / 0 \%-2 \%$ (gastrointestinal perforation). Other than hypertension and proteinuria, these adverse events are often reported as adverse events resulting from glioblastoma itself or its complications; thus, these adverse events are often likely to occur during BV administration. It is necessary to explain to patients the possibility that these adverse events may occur as complications of glioblastoma during BV administration, and patients should be fully briefed on the importance of BV therapy and its asscociated adverse events.

Glioblastoma, like other brain tumors, can cause tumor hemorrhage with an incidence of $\sim 2 \%{ }^{1,56}$ Khasraw et al reported no significant difference in incidence of intracerebral hemorrhage between patients with glioblastoma or metastatic brain tumors treated with and without BV (3.7\% vs 3.6\%). ${ }^{57}$

Up to $20 \%$ and $30 \%$ of patients with glioblastoma were reported to experience venous thrombosis in the perioperative period and during chemotherapy, respectively, as PS is lowered due to paralysis. ${ }^{58}$ Nalluri et al indicated that $\mathrm{BV}$ increased the risk of venous thrombosis by 1.3 -fold in patients with cancer; thus, patients with glioblastoma should receive anticoagulant therapy. ${ }^{59}$ If $\mathrm{BV}$ is administered to patients with glioblastoma with a high risk of cerebral hemorrhage while providing anticoagulant therapy, further increases in the risk of cerebral hemorrhage is a concern. Although previous reports have indicated that BV does not contribute to the incidence of intracerebral hemorrhage while patients are receiving anticoagulant therapy, ${ }^{60,61}$ caution is necessary because an intracerebral hemorrhage is often a life-threatening event.

Administration of BV doubled the risk of cerebral infarction or myocardial infarction compared to patients who did not receive BV. ${ }^{62,63}$ The incidence of cerebral infarction was reported to be $\sim 9 \%$ in patients with glioma because of surgery, chemotherapy, or radiation therapy. ${ }^{64}$ Fraum et al reported no difference in the incidence of cerebral infarction with BV or without BV. ${ }^{56}$

Hypertension is a common adverse event of BV. In their meta-analysis, Ranpura et al reported that the incidence of hypertension due to $\mathrm{BV}$ was $23.6 \%$ and the incidence of grade $3 / 4$ severe hypertension was $7.9 \% .{ }^{65} \mathrm{BV}$ may cause hypertension because of glomerular injury due to occlusion of microarteries in the kidney or a reduction in the production of nitric oxide in the arterial wall. ${ }^{66}$ Some reports confirm the correlation between the administration of BV for glioblastoma and hypertension, ${ }^{67-69}$ while at least one report refutes it. ${ }^{70}$ Thus, the issue remains unresolved.

Proteinuria is an adverse event caused by impairment of glomerular endothelial cells due to $\mathrm{BV} .{ }^{71}$ Regular urinalysis during outpatient visits is essential since proteinuria may lead to nephrotic syndrome or renal failure. According to the meta-analysis conducted by Wu et al, the incidence of grade $3 / 4$ severe proteinuria was $2.2 \% .^{72}$ Both proteinuria and hypertension have been reported to occur even under low-dose administration of BV $(2.5 \mathrm{mg} / \mathrm{kg})$.

Clark et al reported a high complication rate (35\%) in patients undergoing craniotomy after BV therapy, including delayed wound healing and infection, along with an increased risk of delayed wound healing in patients undergoing craniotomy within 28 days of $\mathrm{BV}$ administration. ${ }^{73}$ Abrams analyzed a number of papers to examine an association 
between administration of BV and wound healing, and reported it was necessary to perform craniotomy 4 weeks after cessation of BV therapy, and a 2-week interval after craniotomy is necessary before re-initiation of $\mathrm{BV} .{ }^{74}$

\section{Conclusion}

Although the usefulness of BV administration in patients with newly-diagnosed glioblastoma is partly supported by results such as prolongation of time maintaining KPS $\geq 70$, active administration of $\mathrm{BV}$ at the primary stage remains controversial because optimal treatment after recurrence has not been established. Since the administration of BV at the primary stage failed to prolong survival, $\mathrm{RT}+\mathrm{TMZ}$ remains the standard therapy for glioblastoma. RT $+\mathrm{TMZ}$ therapy should be performed in patients with KPS $\geq 90$, considering the possible adverse events of BV. As most patients with glioblastoma have poor KPS at the primary stage, BV should be administered to patients with poor KPS at initial presentation because BV appears to increase or preserve their PS. Most of the adverse events of BV occur as complications of glioblastoma itself, leading to low PS scores. It is therefore crucial to explain to these patients the advantages and disadvantages of BV administration.

\section{Disclosure}

The author has received speaker honoraria from Chugai Pharmaceutical Co., Merck Sharp and Dohme (MSD), Eisai and Nobelpharma. The author reports no other conflicts of interest in this work.

\section{References}

1. Committee of Brain Tumor Registry of Japan. Report of Brain Tumor Registry of Japan (2001-2004). 13th ed. Neurol Med Chir. 2014; (Suppl 1):55.

2. Stupp R, Hegi ME, Mason WP, et al. Effects of radiotherapy with concomitant and adjuvant temozolomide versus radiotherapy alone on survival in glioblastoma in a randomised phase III study: 5-year analysis of the EORTC-NCIC trial. Lancet Oncol. 2009;10(5):459-466.

3. Stupp R, Mason WP, van den Bent MJ, et al. Radiotherapy plus concomitant and adjuvant temozolomide for glioblastoma. $N$ Engl $\mathrm{J}$ Med. 2005;352(10):987-996.

4. Bates DO. Vascular endothelial growth factors and vascular permeability. Cardiovas Res. 2010;87(2):262-271.

5. Gerstner ER, Duda DG, di Tomaso E, et al. VEGF inhibitors in the treatment of cerebral edema in patients with brain cancer. Nat Rev Clin Oncol. 2009;6(4):229-236.

6. Cohen MH, Shen YL, Keegan P, Pazdur R. FDA drug approval summary: bevacizumab (Avastin) as treatment of recurrent glioblastoma multiforme. Oncologist. 2009;14(11):1131-1138.

7. Takano S, Kimu H, Tsuda K, et al. Decrease in the apparent diffusion coefficient in peritumoral edema for the assessment of recurrent glioblastoma treated by bevacizumab. Acta Neurochir Suppl. 2013;118:185-189.

8. Chinot OL, Wick W, Mason W, et al. Bevacizumab plus radiotherapytemozolomide for newly diagnosed glioblastoma. N Engl J Med. 2014; 370(8):709-722.
9. Vredenburgh JJ, Cloughesy T, Samant M, et al. Corticosteroid use in patients with glioblastoma at first or second relapse treated with bevacizumab in the BRAIN study. Oncologist. 2010;15(12):1329-1334.

10. Nagpal S, Harsh G, Recht L. Bevacizumab improves quality of life in patients with recurrent glioblastoma. Chemother Res Pract. 2011; 2011:602812.

11. Kaley T, Nolan C, Carver A, Omuro A. Bevacizumab for acute neurologic deterioration in patients with glioblastoma. CNS Oncol. 2013; 2(5):413-418.

12. Gilbert MR, Dignam JJ, Armstrong TS, et al. A randomized trial of bevacizumab for newly diagnosed glioblastoma. N Engl J Med. 2014; 370(8):699-708

13. Colman H, Zhang L, Sulman EP, et al. A multigene predictor of outcome in glioblastoma. Neuro Oncol. 2010;12(1):49-57.

14. Taphoorn MJ, Henriksson R, Bottomley A, et al. Health-Related Quality of Life in a Randomized Phase III Study of Bevacizumab, Temozolomide, and Radiotherapy in Newly Diagnosed Glioblastoma. J Clin Oncol. 2015;33(19):2166-2175.

15. Wefel JS, Pugh SL, Armstrong TS, et al. Neurocognitive function (NCF) outcomes in patients with glioblastoma (GBM) enrolled in RTOG 0825. J Clin Oncol. 2013 ASCO Annual Meeting Abstracts. 2013; 31(15 suppl):2004.

16. Armstrong TS, Won M, Wefel JS, et al. Comparative impact of treatment on patient reported outcomes (PROs) in patients with glioblastoma (GBM) enrolled in RTOG 0825. J Clin Oncol. 2013, ASCO Annual Meeting Abstracts. 2013;31(15 suppl):2003.

17. Phillips HS, Kharbanda S, Chen R, et al. Molecular subclasses of highgrade glioma predict prognosis, delineate a pattern of disease progression, and resemble stages in neurogenesis. Cancer Cell. 2006;9(3): 157-173.

18. Sandmann T, Bourgon R, Garcia J, et al. Patients With Proneural Glioblastoma May Derive Overall Survival Benefit From the Addition of Bevacizumab to First-Line Radiotherapy and Temozolomide: Retrospective Analysis of the AVAglio Trial. J Clin Oncol. 2015;33(25):2735-2744.

19. Wick W, Chinot OL, Mason WP, et al. Patterns of tumor progression in a phase 3 study of bevacizumab (Bv) plus radiotherapy (RT) plus temozolomide (T) for newly diagnosed glioblastoma (GB). J Clin Oncol. 2014 ASCO Annual Meeting Abstracts. 2014;32(15 suppl):2014:2051.

20. Vredenburgh JJ, Desjardins A, Herndon JE 2nd, et al. Bevacizumab plus irinotecan in recurrent glioblastoma multiforme. J Clin Oncol. 2007; 25(30):4722-4729.

21. Friedman HS, Prados MD, Wen PY, et al. Bevacizumab alone and in combination with irinotecan in recurrent glioblastoma. J Clin Oncol. 2009;27(28):4733-4740.

22. Kreisl TN, Kim L, Moore K, et al. Phase II trial of single-agent bevacizumab followed by bevacizumab plus irinotecan at tumor progression in recurrent glioblastoma. J Clin Oncol. 2009;27(5):740-745.

23. Chamberlain MC, Johnston SK. Salvage therapy with single agent bevacizumab for recurrent glioblastoma. $J$ Neurooncol. 2010;96(2): 259-269.

24. Kreisl TN, Zhang W, Odia Y, et al. A phase II trial of single-agent bevacizumab in patients with recurrent anaplastic glioma. Neuro Oncol. 2011; 13(10):1143-1150.

25. Nagane M, Nishikawa R, Narita Y, et al. Phase II study of single-agent bevacizumab in Japanese patients with recurrent malignant glioma. Jpn J Clin Oncol. 2012;42(10):887-895.

26. Kaloshi G, Brace G, Rroji A, et al. Bevacizumab alone at $5 \mathrm{mg} / \mathrm{kg}$ in an every-3-week schedule for patients with recurrent glioblastomas: a single center experience. Tumori. 2013;99(5):601-603.

27. Narita Y. Drug review: safety and efficacy of bevacizumab for glioblastoma and other brain tumors. Jpn J Clin Oncol. 2013;43(6):587-595.

28. Wefel JS, Cloughesy T, Zazzali JL, et al. Neurocognitive function in patients with recurrent glioblastoma treated with bevacizumab. Neuro Oncol. 2011;13(6):660-668.

29. Duerinck J, Clement PM, Bouttens F, et al. Patient outcome in the Belgian medical need program on bevacizumab for recurrent glioblastoma. J Neurol. 2015;262(3):742-751. 
30. Tabouret E, Barrie M, Thiebaut A, et al. Limited impact of prognostic factors in patients with recurrent glioblastoma multiforme treated with a bevacizumab-based regimen. J Neurooncol. 2013; 114(2):191-198.

31. Levin VA, Mendelssohn ND, Chan J, et al. Impact of bevacizumab administered dose on overall survival of patients with progressive glioblastoma. J Neurooncol. 2015;122(1):145-150.

32. Lorgis V, Maura G, Coppa G, et al. Relation between bevacizumab dose intensity and high-grade glioma survival: a retrospective study in two large cohorts. J Neurooncol. 2012;107(2):351-358.

33. Taal W, Oosterkamp HM, Walenkamp AM, et al. Single-agent bevacizumab or lomustine versus a combination of bevacizumab plus lomustine in patients with recurrent glioblastoma (BELOB trial): a randomised controlled phase 2 trial. Lancet Oncology. 2014;15(9):943-953.

34. Verhoeff JJ, Lavini C, van Linde ME, et al. Bevacizumab and doseintense temozolomide in recurrent high-grade glioma. Ann Oncol. 2010; 21(8):1723-1727.

35. Desjardins A, Reardon DA, Coan A, et al. Bevacizumab and daily temozolomide for recurrent glioblastoma. Cancer. 2012;118(5):1302-1312.

36. Rahman R, Hempfling K, Norden AD, et al. Retrospective study of carmustine or lomustine with bevacizumab in recurrent glioblastoma patients who have failed prior bevacizumab. Neuro Oncol. 2014;16(11):1523-1529.

37. Liu Z, Zhang G, Zhu L, et al. Retrospective analysis of bevacizumab in combination with fotemustine in Cinese patients with recurrent glioblastoma multiforme. BioMed Res Inter. 2015;2015:723612.

38. Soffietti R, Trevisan E, Bertero L, et al. Bevacizumab and fotemustine for recurrent glioblastoma: a phase II study of AINO (Italian Association of Neuro-Oncology). J Neurooncol. 2014;116(3):533-541.

39. Zhang G, Huang S, Wang Z. A meta-analysis of bevacizumab alone and in combination with irinotecan in the treatment of patients with recurrent glioblastoma multiforme. J Clin Neurosci. 2012;19(12):1636-1640.

40. Field KM, Simes J, Nowak AK, et al. Randomized phase 2 study of carboplatin and bevacizumab in recurrent glioblastoma. Neuro Oncol. 2015;17(11): 1504-1513

41. Kaloshi G, Diamandi P, Cakani B, Brace G, Rroji A, Petrela M. The added value of bevacizumab concomitantly administered with carboplatin versus carboplatin alone in patients with recurrent glioblastomas. Tumori. 2015;101(1):41-45.

42. Reardon DA, Desjardins A, Peters KB, et al. Phase II study of carboplatin, irinotecan, and bevacizumab for bevacizumab naive, recurrent glioblastoma. J Neurooncol. 2012;107(1):155-164.

43. Levin VA, Bidaut L, Hou P, et al. Randomized double-blind placebo-controlled trial of bevacizumab therapy for radiation necrosis of the central nervous system. Int J Radiat Oncol Biol Phys. 2011;79(5): 1487-1495.

44. Back M, Gzell CE, Kastelan M, Guo L, Wheeler HR. Large volume re-irradiation with bevacizumab is a feasible salvage option for patients with refractory high-grade glioma. Neurooncol Pract. 2015; 2(1):48-53.

45. Magnuson W, Ian Robins H, Mohindra P, Howard S. Large volume reirradiation as salvage therapy for glioblastoma after progression on bevacizumab. J Neurooncol. 2014;117(1):133-139.

46. Flieger M, Ganswindt U, Schwarz SB, et al. Re-irradiation and bevacizumab in recurrent high-grade glioma: an effective treatment option. J Neurooncol. 2014;117(2):337-345.

47. Hundsberger T, Brugge D, Putora PM, Weder P, Weber J, Plasswilm L. Re-irradiation with and without bevacizumab as salvage therapy for recurrent or progressive high-grade gliomas. J Neurooncol. 2013;112(1): 133-139.

48. Stupp R, Wong ET, Kanner AA, et al. NovoTTF-100A versus physician's choice chemotherapy in recurrent glioblastoma: a randomised phase III trial of a novel treatment modality. Eur J Cancer. 2012; 48(14):2192-2202.

49. Hamza MA, Mandel JJ, Conrad CA, et al. Survival outcome of early versus delayed bevacizumab treatment in patients with recurrent glioblastoma. J Neurooncol. 2014;119(1):135-140.
50. Piccioni DE, Lai A. Deferred use of bevacizumab for recurrent glioblastoma is not associated with diminished efficacy. Neuro Oncol. 2014;16(10):1427-1428.

51. Zuniga RM, Torcuator R, Jain R, et al. Rebound tumour progression after the cessation of bevacizumab therapy in patients with recurrent high-grade glioma. J Neurooncol. 2010;99(2):237-242.

52. Cohn AL, Bekaii-Saab T, Bendell JC, et al. Clinical outcomes in bevacizumab (BV)-treated patients (pts) with metastatic colorectal cancer (mCRC): results from ARIES observational cohort study (OCS) and confirmation of BRiTE data on BV beyond progression (BBP). J Clin Oncol. 2010 ASCO Annual Meeting Proceedings; 2010;18(15 suppl):3596.

53. Grothey A, Sugrue MM, Purdie DM, et al. Bevacizumab beyond first progression is associated with prolonged overall survival in metastatic colorectal cancer: results from a large observational cohort study (BRiTE). J Clin Oncol. 2008;26(33):5326-5334.

54. Reardon DA, Herndon JE 2nd, Peters KB, et al. Bevacizumab continuation beyond initial bevacizumab progression among recurrent glioblastoma patients. Br J Cancer. 2012;107(9):1481-1487.

55. Hovey EJ, Field KM, Rosenthal MA, et al. Continuing or ceasing bevacizumab at disease progression: results from the CABARET study, a prospective randomized phase II trial in patients with recurrent glioblastoma. J Clin Oncol. 2013 ASCO Annual Meeting Abstracts. 2015;33(15 suppl):2015:2003.

56. Fraum TJ, Kreisl TN, Sul J, Fine HA, Iwamoto FM. Ischemic stroke and intracranial hemorrhage in glioma patients on antiangiogenic therapy. J Neurooncol. 2011;105(2):281-289.

57. Khasraw M, Holodny A, Goldlust SA, DeAngelis LM. Intracranial hemorrhage in patients with cancer treated with bevacizumab: the Memorial Sloan-Kettering experience. Ann Oncol. 2012;23(2):458-463.

58. Perry JR. Thromboembolic disease in patients with high-grade glioma. Neuro Oncol. 2012;14 Suppl 4:iv73-iv80.

59. Nalluri SR, Chu D, Keresztes R, et al. Risk of venous thromboembolism with the angiogenesis inhibitor bevacizumab in cancer patients: a meta-analysis. JAMA. 2008;300(19):2277-2285.

60. Nghiemphu PL, Green RM, Pope WB, Lai A, Cloughesy TF. Safety of anticoagulation use and bevacizumab in patients with glioma. Neuro Oncol. 2008;10(3):355-360.

61. Norden AD, Bartolomeo J, Tanaka S, et al. Safety of concurrent bevacizumab therapy and anticoagulation in glioma patients. J Neurooncol. 2012;106(1):121-125.

62. Ranpura V, Hapani S, Chuang J, Wu S. Risk of cardiac ischemia and arterial thromboembolic events with the angiogenesis inhibitor bevacizumab in cancer patients: a meta-analysis of randomized controlled trials. Acta Oncol. 2010;49(3):287-297.

63. Scappaticci FA, Skillings JR, Holden SN, et al. Arterial thromboembolic events in patients with metastatic carcinoma treated with chemotherapy and bevacizumab. J Natl Cancer Inst. 2007;99(16):1232-1239.

64. Kreis1 TN, Toothaker T, Karimi S, DeAngelis LM. Ischemic stroke in patients with primary brain tumors. Neurology. 2008;70(24): 2314-2320.

65. Ranpura V, Pulipati B, Chu D, Zhu X, Wu S. Increased risk of highgrade hypertension with bevacizumab in cancer patients: a metaanalysis. Am J Hypertens. 2010;23(5):460-468.

66. Izzedine H, Ederhy S, Goldwasser F, et al. Management of hypertension in angiogenesis inhibitor-treated patients. Ann Oncol. 2009; 20(5):807-815.

67. Zhong J, Ali AN, Voloschin AD, et al. Bevacizumab-induced hypertension is a predictive marker for improved outcomes in patients with recurrent glioblastoma treated with bevacizumab. Cancer. 2015; 121(9):1456-1462.

68. Wagner CC, Held U, Kofmehl R, Battegay E, Zimmerli L, Hofer S. Role of arterial hypertension as a predictive marker for bevacizumab efficacy in recurrent glioblastoma - a prospective analysis. Acta Oncol. 2014;53(4):572-575.

69. Mir O, Coriat R, Cabanes L, et al. An observational study of bevacizumab-induced hypertension as a clinical biomarker of antitumor activity. Oncologist. 2011;16(9):1325-1332. 
70. Wick A, Schafer N, Dorner N, et al. Arterial hypertension and bevacizumab treatment in glioblastoma: no correlation with clinical outcome. J Neurooncol. 2010;97(1):157-158.

71. Eremina V, Jefferson JA, Kowalewska J, et al. VEGF inhibition and renal thrombotic microangiopathy. $N$ Engl J Med. 2008;358(11): 1129-1136.

72. Wu S, Kim C, Baer L, Zhu X. Bevacizumab increases risk for severe proteinuria in cancer patients. J Am Soc Nephrol. 2010;21(8): 1381-1389.
73. Clark AJ, Butowski NA, Chang SM, et al. Impact of bevacizumab chemotherapy on craniotomy wound healing. J Neurosurg. 2011; 114(6):1609-1616.

74. Abrams DA, Hanson JA, Brown JM, Hsu FP, Delashaw JB Jr, Bota DA. Timing of surgery and bevacizumab therapy in neurosurgical patients with recurrent high grade glioma. J Clin Neurosci. 2015;22(1): 35-39.

\section{Publish your work in this journal}

Therapeutics and Clinical Risk Management is an international, peerreviewed journal of clinical therapeutics and risk management, focusing on concise rapid reporting of clinical studies in all therapeutic areas, outcomes, safety, and programs for the effective, safe, and sustained use of medicines. This journal is indexed on PubMed Central, CAS,
EMBase, Scopus and the Elsevier Bibliographic databases. The manuscript management system is completely online and includes a very quick and fair peer-review system, which is all easy to use. Visit http://www.dovepress.com/testimonials.php to read real quotes from published authors.

Submit your manuscript here: http://www.dovepress.com/therapeutics-and-clinical-risk-management-journal 